\title{
Tubeimoside I induces autophagy in HepG2 cells by activating the AMP-activated protein kinase signaling pathway
}

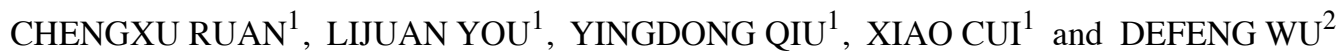 \\ ${ }^{1}$ College of Biological Science and Engineering, Fuzhou University, Fuzhou, Fujian 350116; \\ ${ }^{2}$ College of Bee Science, Fujian Agriculture and Forestry University, Fuzhou, Fujian 350002, P.R. China
}

Received June 22, 2019; Accepted April 1, 2020

DOI: 10.3892/ol.2020.11604

\begin{abstract}
Tubeimoside I (TBMS) is a natural compound with antitumor properties. However, the detailed mechanism underlying the function of TBMS in liver cancer has not been fully elucidated. In the present study, TBMS was shown to suppress cell proliferation and induce $S$ phase cell cycle arrest in HepG2 cells. Furthermore, TBMS treatment induced autophagy, evidenced by autophagosome accumulation, and increased the mRNA expression of Beclin 1 and microtubule-associated protein 1 light chain 3 (LC3)-I. However, flow cytometry analysis demonstrated that TBMS exerted no effect on cell apoptosis. Moreover, TBMS increased the phosphorylation of AMP-activated protein kinase (AMPK) in a concentration-dependent manner, thereby activating the AMPK signaling pathway. A specific AMPK inhibitor, compound C (CC), caused markedly suppressed TBMS-induced accumulation of LC3-II. In addition, the mRNA expression of LC3-I and Beclin 1 was also suppressed in cells treated with TBMS and $\mathrm{CC}$ in combination. The results of the present study provide new insights into the role of TBMS in inducing autophagy and support the potential application of TBMS for liver cancer treatment in the clinical setting.
\end{abstract}

\section{Introduction}

Liver cancer is the third most common cause of cancer-associated mortality and the sixth most common type of human cancer worldwide according to the global cancer statistic report in 2020 (1). The prognosis of patients diagnosed with liver cancer is very poor, due to the severity of the disease (2). Local ablation therapy and surgical resection are only effective at the early stages of liver cancer, and the disease recurs in the majority of patients within 5 years. Other than sorafenib, there

Correspondence to: Professor Defeng Wu, College of Bee Science, Fujian Agriculture and Forestry University, 15 Shangxiadian Road, Cangshan, Fuzhou, Fujian 350002, P.R. China

E-mail: defengwu@yeah.net

Key words: tubeimoside I, liver cancer, autophagy, AMP-activated protein kinase are no effective chemotherapy for advanced disease (3). One of the limitations of anticancer therapy is that a small number of residual tumor cells survive, via autophagy, and repopulate in the host in the absence of stressors. However, molecular targeted therapy may be a potential method for prolonging the survival of patients with advanced liver cancer.

Recent studies have indicated that the autophagic process represents a crucial anticancer mechanism, and enhancing autophagy may be an important therapeutic approach to liver cancer (4-6). The process of autophagy involves the formation of double-membrane autophagosomes, followed by fusion of the autophagosomes with lysosomes to form autolysosomes, and degradation of the contents by lysosomal hydrolases (7). Autophagy may play a dual role in tumors; although it may inhibit tumor progression by degrading oncogenic proteins, autophagy may also assist tumor cells to overcome metabolic stress (8). Several anticancer drugs induce autophagy; however, it remains unclear whether autophagy leads to therapeutic resistance or enhances the antitumor activity of the drugs (9). TubeimosideI(TBMS) is a triterpenoid saponin extracted from the tubers of Bolbostemma paniculatum. The sugar chains of TBMS are linked by 3-hydroxy-3-methylglutaric acid to form a distinct macrocyclic structure (10). TBMS has been shown to induce apoptosis in a variety of human cancer cell lines $(11,12)$. However, the exact effect of TBMS on liver cancer cells remains unclear and the underlying mechanism has yet to be elucidated.

The aim of the present study was to investigate the mechanisms underlying the potent antitumor properties of TBMS, by examining the role of TBMS in cell cycle progression and autophagy in HepG2 cells, in order to determine whether its potential as an alternative therapeutic strategy for liver cancer.

\section{Materials and methods}

Cell culture and drug treatment. HepG2 cells (cat. no. 72) were obtained from the National Infrastructure of Cell Line Resource (Beijing, China) and were authenticated using the STR profiling method. The cells were cultured in Dulbecco's modified Eagle's medium supplemented with $10 \%$ fetal bovine serum (both from Gibco; Thermo Fisher Scientific, Inc.) and incubated at $37^{\circ} \mathrm{C}$ in an atmosphere of $5 \% \mathrm{CO}_{2}$ in air. The cells were seeded at a density of $2 \times 10^{5}$ cells, $24 \mathrm{~h}$ prior to drug treatment. TBMS or Dorsomorphin [compound C (CC)] 
(both purchased from Beyotime Institute of Biotechnology) was dissolved in DMSO and the final concentration of DMSO was maintained at $0.1 \%$. For TBMS treatment, the indicated concentration of TBMS was added to the cells for $24 \mathrm{~h}$. For CC treatment, $4 \mu \mathrm{M}$ of CC was treated alone or together with TBMS for 24 h. Subsequently, the cells were harvested for analysis.

Cell viability assay. Cell viability was assessed by the Cell Counting Kit-8 (CCK-8, Beyotime Institute of Biotechnology) assay. In brief, HepG2 cells were seeded at a density of 2,000 cells/well in 96-well plates. The cells were untreated [negative control (NC) group] or treated with TBMS at the indicated concentrations $(0,0.4,1,2,4,8,12$ and $16 \mu \mathrm{M})$. Cells in the $0 \mu \mathrm{M}$ group were only treated with DMSO and no TMBS. At 12, 24, 48 and $72 \mathrm{~h}, 10 \mu \mathrm{l}$ of the kit reagent was added to each well and incubated at $37^{\circ} \mathrm{C}$ for 2 h. Finally, all plates were scanned by a microplate reader at $450 \mathrm{~nm}$. Cell viability was calculated on the basis of the absorption value.

$R N A$ extraction and reverse transcription-quantitative PCR $(R T-q P C R)$ analysis. Total RNA was extracted using TRIzol ${ }^{\circledR}$ reagent (Invitrogen; Thermo Fisher Scientific, Inc.). RNA $(1 \mu \mathrm{g})$ was then reverse transcribed to produce first-strand cDNA using the PrimeScript RT Reagent kit (Takara Bio, Inc.), following the manufacturer's instructions: Incubation at $42^{\circ} \mathrm{C}$ for $60 \mathrm{~min}$ and $95^{\circ} \mathrm{C}$ for $5 \mathrm{~min}$. cDNA was then used as the template for qPCR using specific primers for Beclin 1, microtubule-associated protein 1 light chain 3 (LC3)-I, p53 and GAPDH. qPCR was conducted for 40 cycles, with $30 \mathrm{sec}$ of denaturation at $95^{\circ} \mathrm{C}$, and $1 \mathrm{~min}$ of annealing and extension at $60^{\circ} \mathrm{C}$. qPCR was performed with the SYBR Green qPCR Master Mix (Takara Bio, Inc.) on IQ5 machine (Bio-Rad Laboratories, Inc.). The analysis of gene expression was performed by Bio-Rad Software Manager, version 1.5 (Bio-Rad Laboratories, Inc.). The quantification of relative expression was performed using the $2^{\Delta \Delta \mathrm{Cq}}$ method (13). The primers for qPCR were listed as follows: Beclin-1, forward: 5'-ACCGTGTCACCATCCAGGAA-3' and reverse: 5'-GAA GCTGTTGGCACT TTCTGT-3'; LC3, forward: 5'-GATGTC CGACTTATTCGAAGC-3' and reverse: 5'-TTGAGCTGT AAGCGCCTTCTA-3'; p53, forward: 5'-AGAGTCTATAGG CCC ACCCC-3' and reverse: 5'-GCTCGACGCTAGGATCTG AC-3'; GAPDH, forward: 5'-GAAATCCCATCACCATCT TCCAGG-3' and reverse 5'-GAGCCCCAGCCTTCTCCA TG-3'.

Western blot analysis. Cells were lysed with lysis buffer (120 mmol/1 NaCl, 40 mmol/1 Tris- $\mathrm{HCl}, 0.5 \% \mathrm{NP}-40$ ). The protein concentrations were determined using a BCA kit (Beyotime Institute of Biotechnology). Samples (40 $\mu \mathrm{g}$ protein) were subjected to $10 \%$ SDS-PAGE, and then blotted onto PVDF membranes. The membranes were blocked with 5\% skimmed milk at room temperature for $1 \mathrm{~h}$, and then probed with the indicated primary antibodies against p-AMPK (dilution 1:500, cat. no. ab23875, Abcam), AMPK (dilution 1:500, cat. no. ab32047, Abcam), LC3-I (dilution 1:1,000, cat. no. ab128025, Abcam), LC3-II (dilution 1:1,000, cat. no. ab62721, Abcam) or $\beta$-actin (dilution 1:10,000, cat. no. ab8227, Abcam) at $4^{\circ} \mathrm{C}$ overnight. The membranes were washed and incubated with horseradish peroxidase-conjugated goat anti-rabbit secondary antibodies (dilution 1:5,000, cat. no. ab7090, Abcam) at room temperature for $1 \mathrm{~h}$. Images were visualized using the electrochemiluminescence (ECL) kit (Beyotime Institute Biotechnology). Protein expression was normalized to the internal control GAPDH gene expression. The expression of proteins was quantified using densitometry analysis (ChemiDoc XRS+ gel imaging system) and analyzed using ImageLab Software (version 3.0; BioRad Laboratories, Inc.).

Cell cycle analysis. The effect of TBMS on the cell cycle in HepG2 cells was determined by FACSCalibur flow cytometry (Becton Dickinson and Company). Specifically, HepG2 cells were seeded at a density of $2 \times 10^{5}$ cells/well. Following treatment with TBMS, the cells were harvested with trypsinization and fixed with $70 \%$ ethanol overnight at $4^{\circ} \mathrm{C}$. The fixed cells were then resuspended in PBS solution containing $20 \mu \mathrm{g} / \mathrm{ml}$ propidium iodide (PI; Nanjing KeyGen Biotech Co., Ltd.), $0.5 \mathrm{mg} / \mathrm{ml} \mathrm{RNase}$ and $1 \%$ fetal calf serum, and incubated at $37^{\circ} \mathrm{C}$ for $30 \mathrm{~min}$ without light exposure. The cells were finally analyzed using flow cytometry with FlowJo software (version 10, FlowJo, LLC).

Annexin $V$ apoptosis assay. The effect of TBMS on the apoptosis in HepG2 cells was analyzed using flow cytometry. HepG 2 cells were seeded at a density of $2 \times 10^{5}$ cells/ well. Following treatment with TBMS, adherent cells were harvested by trypsinization and combined with suspended cells. Subsequently, the cells were collected and then resuspended in $500 \mu \mathrm{l}$ of $1 \mathrm{X}$ Annexin $\mathrm{V}$ binding buffer $(2.5 \mathrm{mM}$ $\mathrm{CaCl}_{2}, 140 \mathrm{mM} \mathrm{NaCl}$, and $10 \mathrm{mM}$ HEPES). A total of $2 \mu \mathrm{PI}$ $(1 \mathrm{mg} / \mathrm{ml})$ and $5 \mu \mathrm{l}$ FITC-conjugated Annexin V were added to the cells for $15 \mathrm{~min}$ at room temperature. The percentage of FITC-positive cells was analyzed by flow cytometry using FlowJo software version 10 (FlowJo, LLC).

Transmission electron microscopy (TEM). The cells were fixed in $0.1 \mathrm{~mol} / 1$ phosphate buffer $(\mathrm{pH} 7.4)$ containing $2.5 \%$ glutaraldehyde overnight at $4^{\circ} \mathrm{C}$, followed by the addition of $1 \% \mathrm{OsO}_{4}$. Then, the fixed cells were washed with PBS solution, dehydrated through different concentrations $(70,80,90$ and $100 \%$ ) of ethanol at room temperature for $30 \mathrm{~min}$, and embedded in epoxy resin. After dehydration, thin sections $(1 \mathrm{~mm})$ were stained with lead citrate and uranyl acetate for $30 \mathrm{~min}$ at $4^{\circ} \mathrm{C}$, and observed under a transmission electron microscope (100x magnification; JEM-2010; JEOL Ltd.). The images were captured digitally and analyzed by Image Pro Plus7 software (Media Cybernetics, Inc.). The mean number of autophagosomes per cell was determined from a randomly selected pool of 15-20 fields.

Statistical analysis. Data are expressed as the mean \pm standard deviation from three independent experiments. Data analysis was performed using GraphPad Prism 5 software (GraphPad Software, Inc.). One-way ANOVA was used to analyze the comparisons among multiple groups, followed by Tukey's post hoc test for correction. $\mathrm{P}<0.05$ was considered to indicate statistically significant differences. 

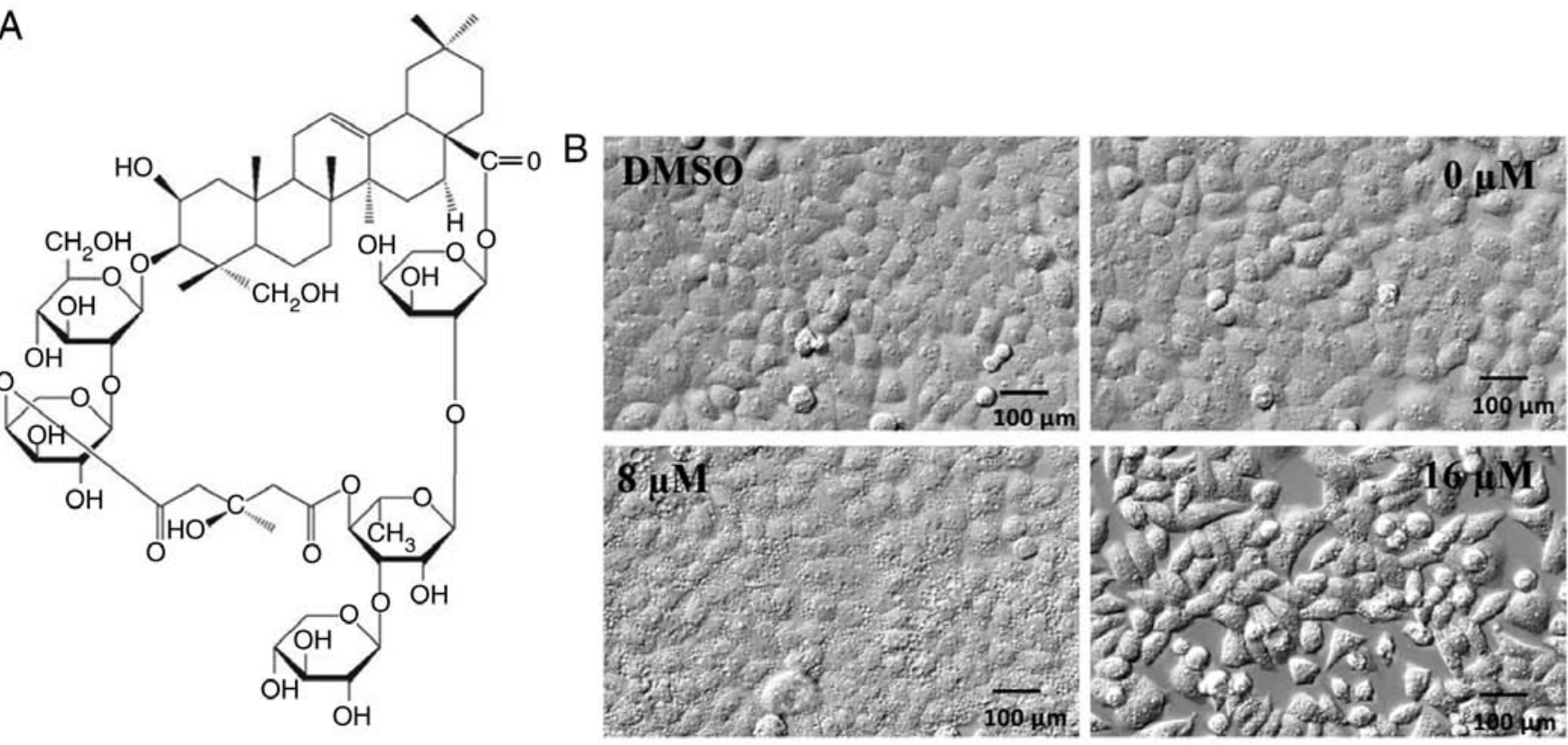

TBMS

Figure 1. TBMS treatment induces cell morphological changes in HepG2 cells. (A) Chemical structure of TBMS. (B) Morphological changes in HepG2 cells in response to TBMS treatment. Bright field mode images of cells after treatment for $24 \mathrm{~h}$ with either DMSO or $0,8,16 \mu \mathrm{M}$ TBMS. TBMS, tubeimoside I.

\section{Results}

TBMS induces extensive intracellular vacuolization and cell morphological changes. The chemical structure of TBMS is presented in Fig. 1A. To examine the changes in cell morphology in response to TBMS exposure, HepG2 liver cancer cells were treated with either DMSO or TBMS at different concentrations for $24 \mathrm{~h}$. The cell morphologies were then captured using an electron microscope (Fig. 1B). Following treatment with DMSO, HepG2 cells exhibited a common epithelial-like shape with adherence. In the presence of $8 \mu \mathrm{M}$ TBMS, HepG2 cells displayed extensive intracellular vacuolization, which was observed as early as $12 \mathrm{~h}$ after drug treatment. Of note, extensive vacuolization of cells may be triggered by a moderate increase in drug concentration. Following treatment with $16 \mu \mathrm{M}$ TBMS, the cell morphology changed and the cells assumed a spindled shape. These results demonstrated that TBMS treatment induced morphological changes and intracellular vacuolization in HepG2 cells.

TBMS causes $S$ phase cell cycle arrest. To investigate the cytotoxic properties of TBMS, the effect of TBMS on HepG2 cell viability was examined using the CCK- 8 assay. HepG2 cells were treated with TBMS at various concentrations $(0$, $0.4,1,2,4,8,12$ and $16 \mu \mathrm{M})$ for different durations $(12,24$, 48 and 72 h). As shown in Fig. 2A, TBMS treatment markedly inhibited cell viability in a concentration-dependent manner. Of note, although the viability of HepG2 cells was concentration-dependently suppressed, with prolonged time, all the cells treated with different concentrations of TBMS survived, although their growth was inhibited. Notably, there were no notable differences among the negative control (NC), $0,0.4,1$ and $2 \mu \mathrm{M}$ TBMS groups (Fig. 2B). Starting from the $4-\mu \mathrm{M}$ group, the cell viability was suppressed by TMBS in a dose-dependent manner. However, when the concentration reached $16 \mu \mathrm{M}$, proliferation was significantly inhibited at $48 \mathrm{~h}$ and did not proliferate at $72 \mathrm{~h}(\mathrm{P}<0.01)$. These results suggested that TBSM caused the cells to lose their proliferative ability.

To further explore the effect of TBMS on cell cycle progression, HepG2 cells were treated with $16 \mu \mathrm{M}$ TBSM or DMSO control for different durations, and flow cytometry analysis was subsequently performed. As shown in Fig. $2 \mathrm{C}$ and D, TBMS treatment led to a time-dependent accumulation of HepG2 cells in the $S$ phase. Subsequently, prolonged treatment of HepG2 cells resulted in a substantial increase in the percentage of S phase cells, from $18.74 \%$ at $24 \mathrm{~h}$ to $32.93 \%$ at $96 \mathrm{~h}$. In addition, the S phase of the NC group exhibited no significant changes at 24 and $96 \mathrm{~h}$. These data suggest that TBMS induces cell cycle arrest at the $\mathrm{S}$ phase.

TBMS induces cell autophagy, but not apoptosis. To determine whether TBMS induces autophagy in HepG2 cells, the cells were treated with TBMS at different concentrations $(8$ and $16 \mu \mathrm{M})$ or DMSO [negative control (NC)] for $24 \mathrm{~h}$. The cytoplasmic accumulation of autophagosomes is generally consistent with the increased expression of biomarkers in the autophagic pathway. Therefore, TEM was first performed to evaluate the morphological changes in the treated cells. As shown in Fig. 3A, there was a higher number of mitochondria around the nucleus in NC cells, indicating that the cells were in an active metabolic state. In comparison, several autolysosomes were observed in the TBMS-treated cells, and the mitochondria were swollen and fewer in number. These results suggested that the cells could be undergoing autophagy. To confirm this hypothesis, the biomarkers of autophagy, Beclin 1 and LC3-I, were detected using RT-qPCR. As shown in Fig. 3B and C, the mRNA expression of Beclin 1 and LC3-I 

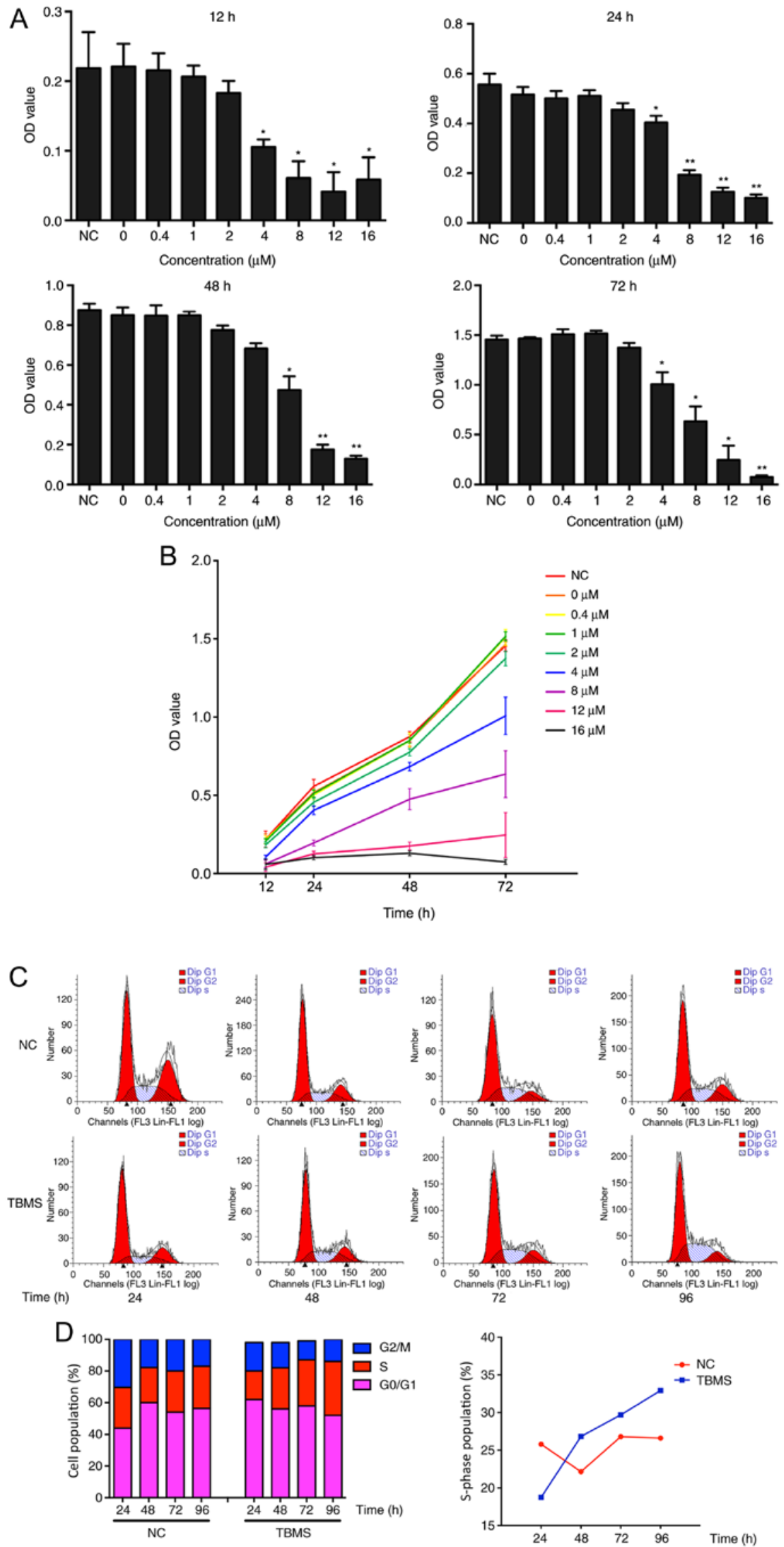

Figure 2. TBMS causes cytotoxicity and S phase cell cycle arrest in HepG2. (A) HepG2 cells were treated with TBMS at various concentrations of $0,0.4,1$, $2,4,8,12$ and $16 \mu \mathrm{M}$ for different durations $\left(12,24,48,72 \mathrm{~h}\right.$ ). The cytotoxicity of TBMS was determined using CCK-8 assay. "P<0.05 and ${ }^{* *} \mathrm{P}<0.01 \mathrm{vs}$. NC. (B) The cells were treated with the different concentration of TBMS for different durations. The cell growth was determined by CCK-8 assay. (C) HepG2 cells were treated with DMSO (NC) or $16 \mu \mathrm{M}$ TBMS for $24,48,72$ and $96 \mathrm{~h}$. The cell cycle distribution was analyzed by flow cytometry. (D) (Left) Quantification of cell cycle distribution after TBMS treatment; (right) the percentage of S phase in cells treated with NC or TBMS for different times. TBMS, tubeimoside I; CCK-8, Cell Counting Kit-8; NC, negative control. 
A

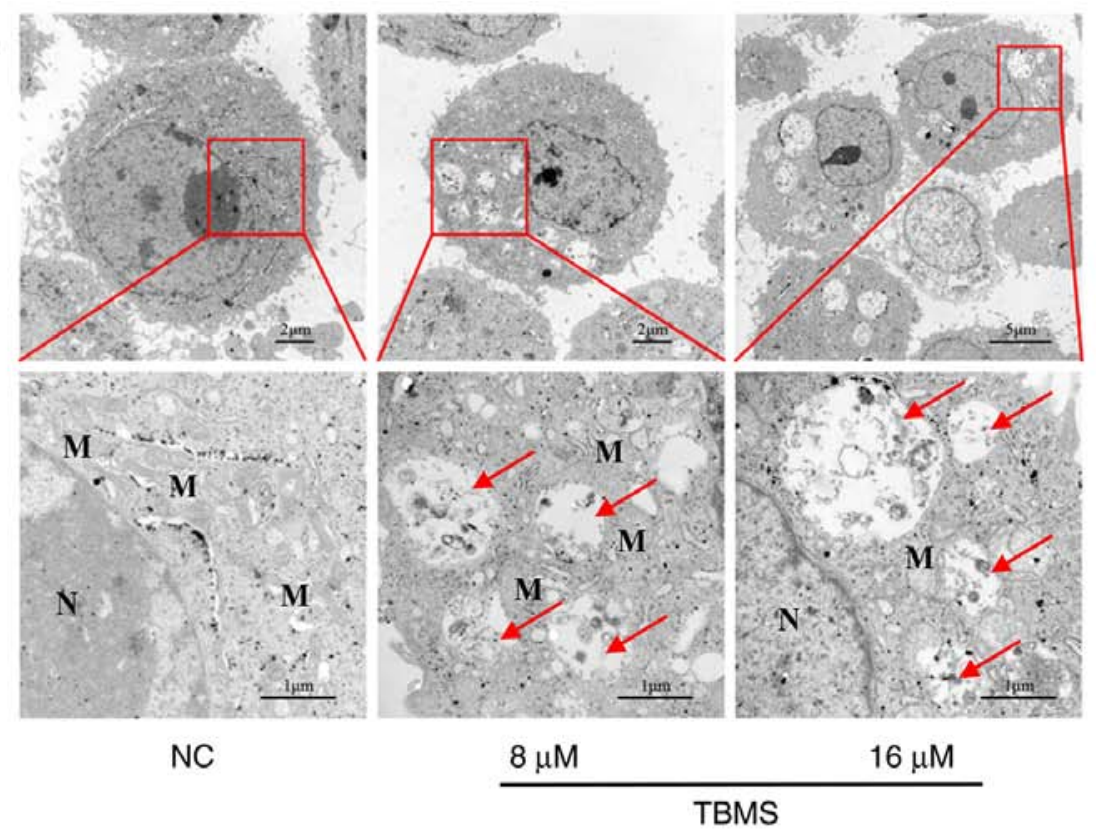

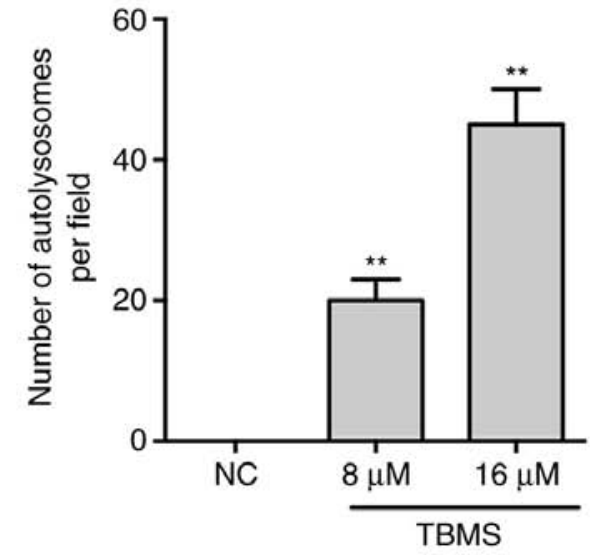

B

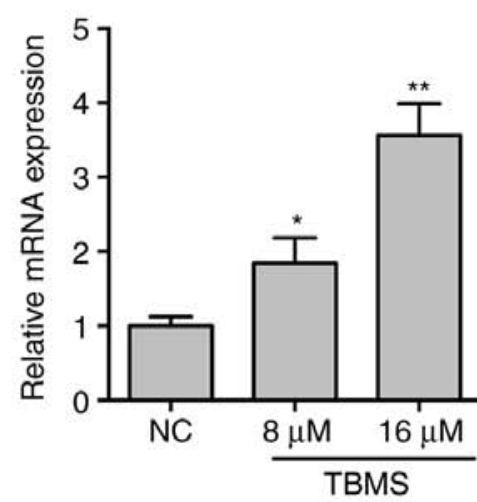

LC3-I

C

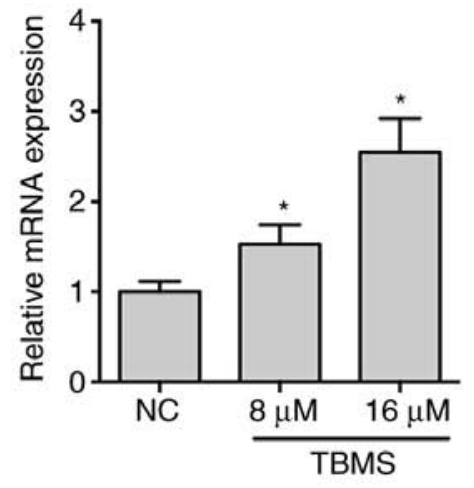

Figure 3. TBMS induces autophagy in HepG2 cells. (A) HepG2 cells were treated with DMSO, 8 or $16 \mu \mathrm{M}$ of TBMS for $24 \mathrm{~h}$. Autolysosomes in cells were observed by transmission electron microscopy and quantified. Red arrows denote autolysosomes. RT-qPCR analysis was performed to detect the mRNA expression of (B) Beclin 1 and (C) LC3-I in cells treated with DMSO, 8 or $16 \mu \mathrm{M}$ of TBMS. "P<0.05 and ${ }^{* *} \mathrm{P}<0.01$ vs. NC. TBMS, tubeimoside I; RT-qPCR, reverse transcription-quantitative PCR; LC3, microtubule-associated protein 1 light chain 3; NC, negative control; N, nucleus; M, mitochondria.

increased with increasing concentration of TBMS, indicating that the cells were indeed undergoing autophagy.

To assess the effect of TBMS on apoptosis, HepG2 cells were treated with different concentrations of TBSM $(0,0.2,2,4,8$ and $16 \mu \mathrm{M})$ for $24 \mathrm{~h}$ (Fig. 4A), or treated with $16 \mu \mathrm{M}$ of TBSM for different durations (24, 48 and 72 h; Fig. 4B). Subsequently, the cells were harvested for apoptosis analysis by flow cytometry. The results demonstrated that TBMS induced neither concentration-dependent nor time-dependent apoptosis significantly. There were no statistical differences among the groups after quantification analysis (Fig. S1). By contrast, doxorubicin, which was used as a positive control, was able to induce apoptosis in HepG2 cells in a dose-dependent manner (Fig. S2). In cells undergoing apoptosis, the upregulation of the p53 gene is considered as an indication of apoptosis (14). The mRNA expression level of p53 was then detected in cells following treatment with different doses of TBMS. The RT-qPCR results demonstrated that the mRNA expression of $\mathrm{p} 53$ decreased with increasing dose of TBMS, confirming that apoptosis did not occur (Fig. S3). Taken together, these results suggest that TBMS induces autophagy; however, not apoptosis in HepG2 cells.

TBMS induces autophagy by activating the AMP-activated protein kinase $(A M P K)$ signaling pathway. The AMPK and mTOR signaling pathways have been reported to be involved in the initiation of autophagy (15). To further explore the molecular mechanism underlying TBMS-induced autophagy, the AMPK expression levels were measured in HepG2 cells following treatment with TBMS. Western blot analysis demonstrated that TBMS treatment led to increased expression of phosphorylated-AMPK (pAMPK) in a dose-dependent manner (Fig. 5A). To further investigate the role of AMPK in TBMS-induced autophagy, CC, a specific AMPK inhibitor, was employed. As shown in Fig. 5B, co-treatment with TBMS and CC markedly suppressed the mRNA expression of Beclin 1 and LC3-I. During autophagosome formation, free 
A

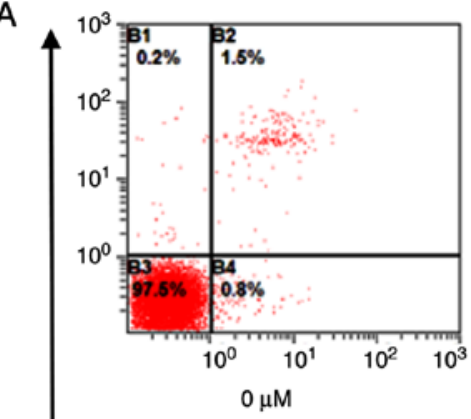

$\bar{\alpha}$

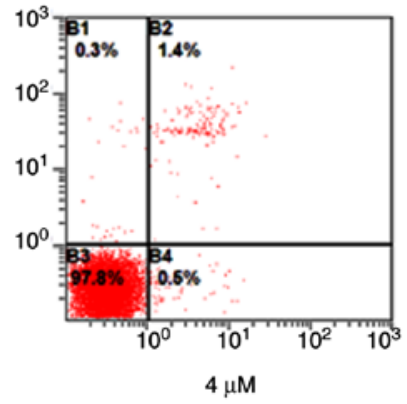

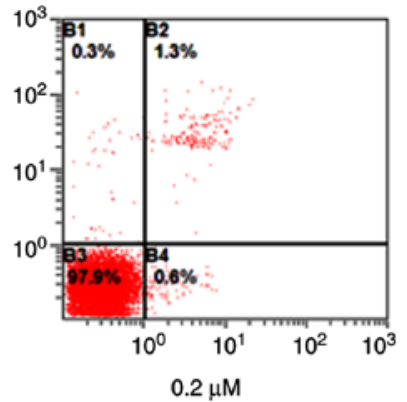

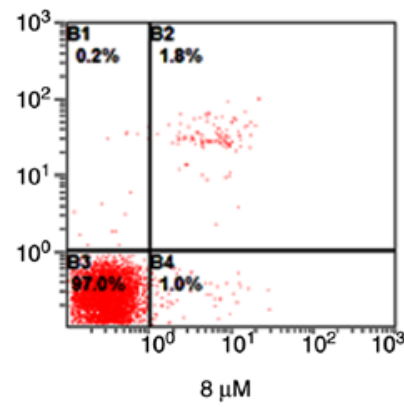

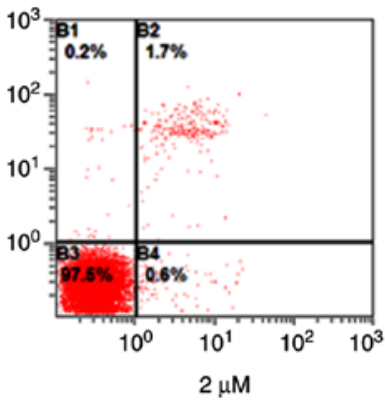

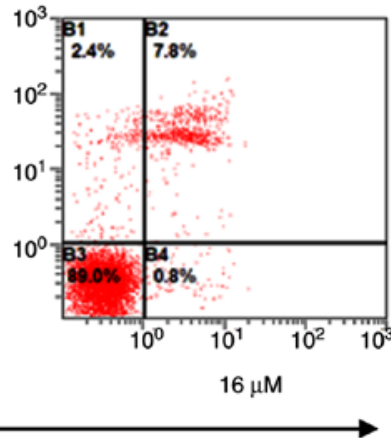

Annexin V-FITC

B

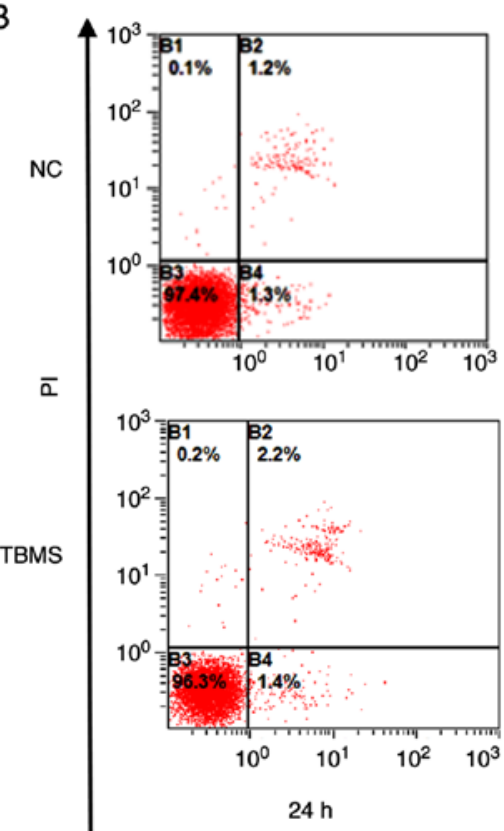

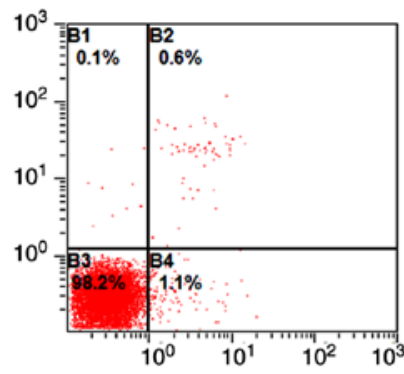
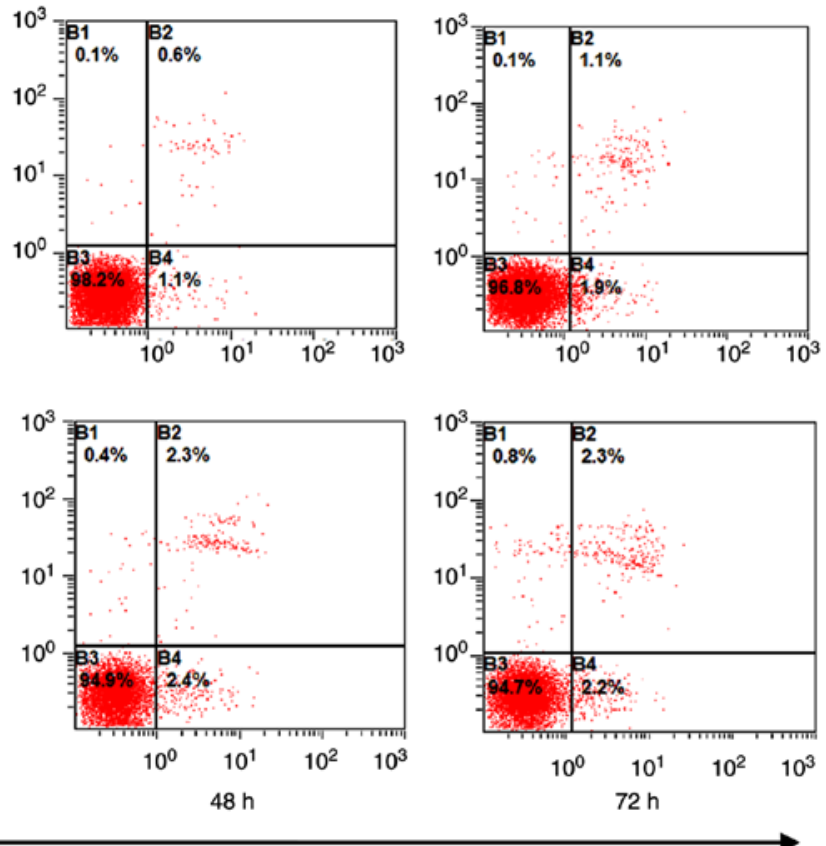

Annexin V-FITC

Figure 4. TBMS does not induce apoptosis in HepG2 cells. (A) HepG2 cells were treated with different concentrations $(0,0.2,2,4,8$ and $16 \mu$ M) of TBMS for $24 \mathrm{~h}$. The cells were then harvested and stained with Annexin V-FITC/PI, and flow cytometry was used to analyze apoptosis. (B) HepG2 cells were treated with DMSO or $16 \mu \mathrm{M}$ TBMS for 24,48 or $72 \mathrm{~h}$. The cells were then harvested and stained with Annexin V/PI, to analyse apoptosis by flow cytometry. TBMS, tubeimoside I; PI, propidium iodide; NC, negative control.

LC3-I in the cytosol is modified and converted to LC3-II, and finally aggregates on the membrane of the autophagosomes. Therefore, the transformation of LC3-I to LC3-II in HepG2 cells was investigated following treatment with TBMS and/or $\mathrm{CC}$. The western blot analysis results demonstrated that $\mathrm{CC}$ significantly decreased TBMS-induced LC3-II accumulation (Fig. 5C and D). Taken together, these results suggest that TBMS induces autophagy by activating the AMPK signaling pathway in HepG2 cells.

\section{Discussion}

The results of the present study may improve the current understanding of the function of TBMS, by uncovering its cellular effects on autophagy in HepG2 cells. HepG2 cells have been proven to be valuable in liver cancer research. Moreover, their ability to genetically modify genes of interest, such as by silencing or overexpression, facilitates in-depth mechanistic studies at the molecular level (16). Although human primary 
B

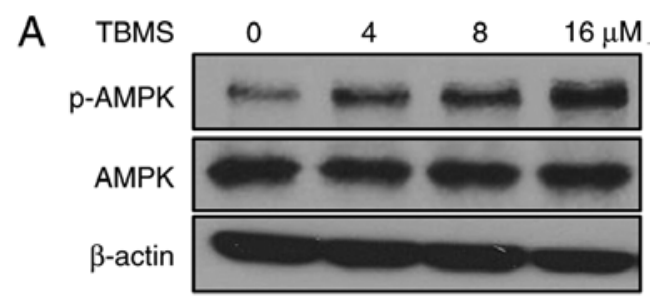

C

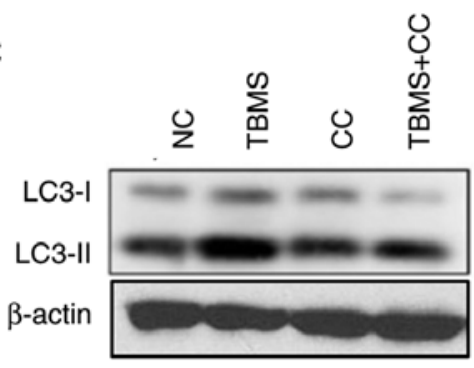

Beclin1

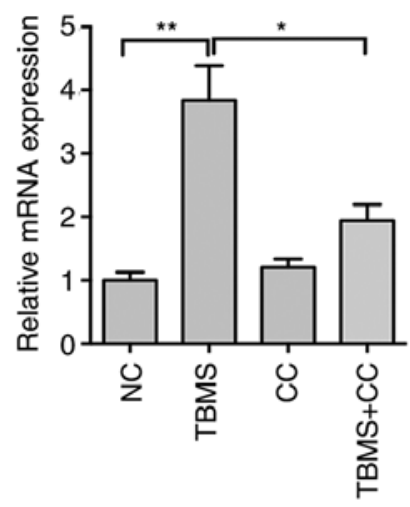

LC3-I

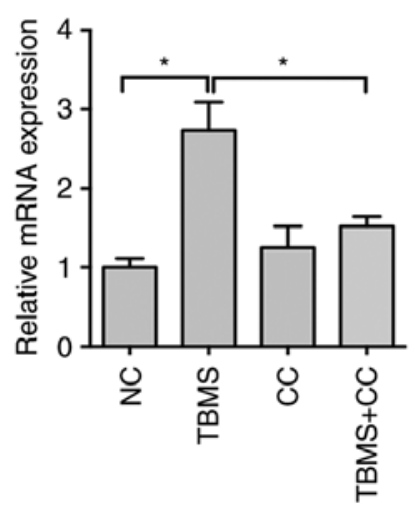

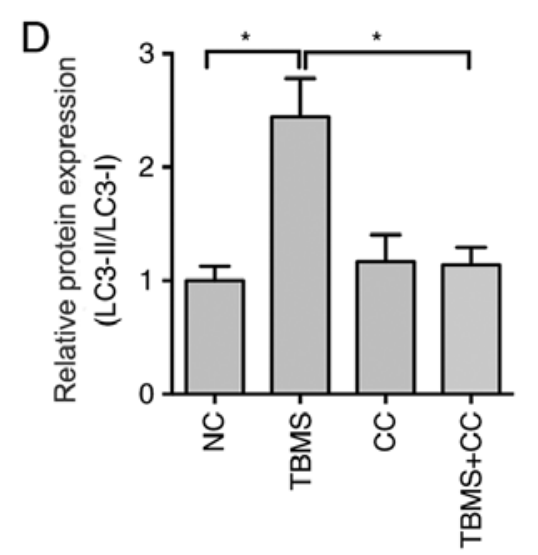

Figure 5. TBMS induces AMPK-dependent autophagy. (A) Western blot analysis of AMPK and AMPK phosphorylation (T172) in HepG2 cells treated with the indicated concentrations of TBMS for $24 \mathrm{~h}$. $\beta$-actin was used as the internal control. (B) HepG 2 cells were treated with TBMS in the absence or presence of CC for $24 \mathrm{~h}$. The mRNA expression of Beclin 1 and LC3-I was measured by RT-qPCR assay. (C) HepG2 cells were treated with TBMS in the absence or presence of CC for $24 \mathrm{~h}$. The protein expression of LC3-I and LC3-II was measured by western blot analysis. $\beta$-actin was used as the internal control. (D) Densitometric analysis of LC3-II/LC3-I is shown in the histogram. The data represent the mean $\pm \mathrm{SD}$. ${ }^{*} \mathrm{P}<0.05,{ }^{* * *} \mathrm{P}<0.01$ vs. NC group. TBMS, tubeimoside I; AMPK, AMP-activated protein kinase; CC, compound C; LC3, microtubule-associated protein 1 light chain 3; RT-qPCR, reverse transcription-quantitative PCR.

hepatocytes may be more relevant, the high variability and limited availability among different donors limit their use (17). Therefore, the HepG2 cell line is a useful model for studying the mechanism of action of anticancer drugs in liver cancer.

Adaptation to the tumor microenvironment (TME) is crucial for tumor cell survival. Metabolic stress is a characteristic of the TME, and most chemotherapeutic agents lead to cellular stress (18). Extracellular stimulation may also induce autophagy; however, its specific function in stress response is not clear. Therefore, whether autophagy-dependent survival should be prevented in these environments to stimulate tumor-cell death is an area that warrants further in-depth investigation. Autophagy plays a complex role in tumorigenesis, since its function in tumor cell death and survival depends on the context. The results of the present study indicated that autophagy is involved in tumor cell survival, particularly as a modulator of the AMPK signaling pathway. The AMPK pathway is considered to be one of the most crucial signaling pathways in human cancer (19). The sustained activation of AMPK regularly occurs in human cancer, and results in a variety of changes to gene expression, involving in cell proliferation, differentiation, metastasis or invasion. The results of the present study demonstrated that TBMS treatment activated the phosphorylation of AMPK dose-dependently, and blocked AMPK function by its specific inhibitor CC suppressed the mRNA expression of Beclin 1 and
LC3-I, indicating that TBMS promotes autophagy by activating the AMPK signaling pathway.

Autophagy, or cellular self-digestion, is a vital cellular recycling mechanism that is responsible for the degradation of malfunctioning or unnecessary cellular proteins and organelles (20). Autophagy is particularly active during metabolic stress. In cancer cells, autophagy plays a dual role as a promoter or inhibitor of tumor growth. Functional autophagy can prevent inflammation and necrosis, which are involved in cell death and result in genetic instability. On the other hand, autophagy provides energy through its circulatory mechanism in an adverse metabolic environment, which may be important for tumor progression (21). Autophagy may be triggered by a wide range of stimuli, and it can induce either cell death or cell survival under different conditions (8). Therefore, studying autophagy may eventually help clinicians and scientists identify methods of interfering with this process, thereby improving human health. Researchers are starting to realize the role of autophagy in drug-induced hepatotoxicity $(22,23)$. LC3 is a biomarker that is widely used for monitoring autophagy (24). To determine whether TBMS treatment can induce autophagy, the activity of autophagy was measured in HepG2 cells. The lipid-conjugated form of LC3 is localized to the membranes of autophagosomes. RT-qPCR revealed that LC3-I was upregulated in TBSM-treated cells, suggesting the 
accumulation of autophagosomes. These changes were verified by electron microscopy and the results demonstrated the different stages of autophagic vacuole formation. These data confirmed that TBSM treatment is accompanied by the activation of autophagy.

In conclusion, the present study demonstrated that TBMS triggered autophagy in HepG2 cells by inducing the accumulation of impaired autophagosomes, and its mechanisms of action may be associated with the activation of the AMPK signaling pathway. Therefore, TBMS is a specific autophagy regulator that may be used as a potential adjuvant for cancer therapy, particularly in the treatment of liver cancer.

\section{Acknowledgements}

Not applicable.

\section{Funding}

This study was supported by Science and Technology Plan Project of Fuzhou (grant no. 2019-G-43).

\section{Availability of data and materials}

The datasets used and/or analyzed during the present study are available from the corresponding author on reasonable request.

\section{Authors' contributions}

CR and DW conceived the study, and analyzed and interpreted the data. CR, LY, YQ and XC performed the experiments and analyzed data. CR wrote the manuscript. DW designed experiments and analyzed the data. All authors read and approved the final manuscript.

\section{Ethics approval and consent to participate}

Not applicable.

\section{Patient consent for publication}

Not applicable.

\section{Competing interests}

The authors declare that they have no competing interests.

\section{References}

1. Siegel RL, Miller KD and Jemal A: Cancer Statistics, 2020. CA Cancer J Clin: 70: 7-30, 2020.

2. Tejeda-Maldonado J, García-Juárez I, Aguirre-Valadez J, González-Aguirre A, Vilatobá-Chapa M, Armengol-Alonso A, Escobar-Penagos F, Torre A, Sánchez-Ávila JF and Carrillo-Pérez DL: Diagnosis and treatment of hepatocellular carcinoma: An update. World J Hepatol 7: 362-376, 2015.

3. Gomaa AI and Waked I: Recent advances in multidisciplinary management of hepatocellular carcinoma. World J Hepatol 7: 673-687, 2015.
4. Janku F, McConkey DJ, Hong DS and Kurzrock R: Autophagy as a target for anticancer therapy. Nat Rev Clin Oncol 8: 528-539, 2011.

5. Wang FZ, Xing L, Tang ZH, Lu JJ, Cui PF, Qiao JB, Jiang L, Jiang HL and Zong L: Codelivery of Doxorubicin and shAkt1 by Poly(ethylenimine)-Glycyrrhetinic acid nanoparticles to induce autophagy-mediated liver cancer combination therapy. Mol Pharm 13: 1298-1307, 2016.

6. Tong H, Li T, Qiu W and Zhu Z: Claudin-1 silencing increases sensitivity of liver cancer HepG2 cells to 5 -fluorouracil by inhibiting autophagy. Oncol Lett 18: 5709-5716, 2019.

7. Mizushima N, Yoshimori T and Levine B: Methods in mammalian autophagy research. Cell 140: 313-326, 2010.

8. Baehrecke EH: Autophagy: Dual roles in life and death? Nat Rev Mol Cell Biol 6: 505-510, 2005.

9. Sui X, Chen R, Wang Z, Huang Z, Kong N, Zhang M, Han W, Lou F, Yang J, Zhang Q, et al: Autophagy and chemotherapy resistance: A promising therapeutic target for cancer treatment. Cell Death Dis 4: e838, 2013.

10. Kasai R, Miyakoshi M, Matsumoto K, Nie RL, Zhou J, Morita T and Tanaka O: Tubeimoside I, a new cyclic bisdesmoside from Chinese cucurbitaceous folk medicine 'tu bei mu', a tuber of Bolbostemma paniculatum. Chem Pharm Bull (Tokyo) 34: 3974-3977, 1986.

11. Jia G, Wang Q, Wang R, Deng D, Xue L, Shao N, Zhang Y, Xia X, Zhi F and Yang Y: Tubeimoside-1 induces glioma apoptosis through regulation of $\mathrm{Bax} / \mathrm{Bcl}-2$ and the ROS/Cytochrome C/Caspase-3 pathway. Onco Targets Ther 8: 303-311, 2015.

12. Zhang Y, Xu XM, Zhang M, Qu D, Niu HY, Bai X, Kan L and He P: Effects of tubeimoside-1 on the proliferation and apoptosis of BGC823 gastric cancer cells in vitro. Oncol Lett 5: 801-804, 2013.

13. Livak KJ and Schmittgen TD: Analysis of relative gene expression data using real-time quantitative PCR and the 2(-Delta Delta C(T)) method. Methods 25: 402-408, 2001.

14. Abu-Qare AW and Abou-Donia MB: Biomarkers of apoptosis: Release of cytochrome c, activation of caspase-3, induction of 8-hydroxy-2'-deoxyguanosine, increased 3-nitrotyrosine, and alteration of p53 gene. J Toxicol Environ Health B Crit Rev 4: 313-332, 2001

15. Dikic I and Elazar Z: Mechanism and medical implications of mammalian autophagy. Nat Rev Mol Cell Biol 19: 349-364, 2018.

16. Toh TB, Lim JJ and Chow EK: Epigenetics of hepatocellular carcinoma. Clin Transl Med 8: 13, 2019.

17. Seow TK, Liang RC, Leow CK and Chung MC: Hepatocellular carcinoma: From bedside to proteomics. Proteomics 1: 1249-1263, 2001.

18. Ferro F, Servais S, Besson P, Roger S, Dumas JF and Brisson L: Autophagy and mitophagy in cancer metabolic remodelling. Semin Cell Dev Biol 98: 129-138, 2019.

19. Cheng J, Zhang T, Ji H, Tao K, Guo J and Wei W: Functional characterization of AMP-activated protein kinase signaling in tumorigenesis. Biochim Biophys Acta 1866: 232-251, 2016.

20. Poillet-Perez $L$ and White E: Role of tumor and host autophagy in cancer metabolism. Genes Dev 33: 610-619, 2019.

21. Levine B and Kroemer G: Autophagy in the pathogenesis of disease. Cell 132: 27-42, 2008.

22. Apostolova N, Gomez-Sucerquia LJ, Gortat A, Blas-Garcia A and Esplugues JV: Autophagy as a rescue mechanism in efavirenz-induced mitochondrial dysfunction: A lesson from hepatic cells. Autophagy 7: 1402-1404, 2011.

23. Ni HM, Bockus A, Boggess N, Jaeschke H and Ding WX: Activation of autophagy protects against acetaminophen-induced hepatotoxicity. Hepatology 55: 222-232, 2012.

24. Kabeya Y, Mizushima N, Ueno T, Yamamoto A, Kirisako T, Noda T, Kominami E, Ohsumi Y and Yoshimori T: LC3, a mammalian homologue of yeast Apg8p, is localized in autophagosome membranes after processing. EMBO J 19: 5720-5728, 2000 .

This work is licensed under a Creative Commons Attribution-NonCommercial-NoDerivatives 4.0 International (CC BY-NC-ND 4.0) License. 